\title{
Correction to: Expression of kallikrein-related peptidase 13 is associated with poor prognosis in esophageal squamous cell carcinoma
}

\author{
Kyoko Nohara ${ }^{1}$ Kazuhiko Yamada ${ }^{1}$. Leo Yamada ${ }^{1,5} \cdot$ Teruki Hagiwara $^{2} \cdot$ Toru Igari $^{3} \cdot$ Chizu Yokoi $^{4} \cdot$ Daisuke Soma $^{1}$. \\ Satoshi Yamashita ${ }^{1} \cdot$ Taeko Dohi $^{2} \cdot$ Yuki I. Kawamura ${ }^{2}$
}

Published online: 24 April 2018

(c) The Japanese Association for Thoracic Surgery 2018

\section{Correction to: General Thoracic and Cardiovascular Surgery https://doi.org/10.1007/s11748-018-0910-5}

In the original publication of the article, Fig. 1 was published incorrectly. The correct Fig. 1 is given in the following page.

The original article can be found online at https://doi.org/10.1007/ s11748-018-0910-5.

Yuki I. Kawamura

kawamura@hospk.ncgm.go.jp

1 Department of Surgery, National Center for Global Health and Medicine, Tokyo, Japan

2 Department of Gastroenterology, The Research Center for Hepatitis and Immunology, Research Institute, National Center for Global Health and Medicine, 1-7-1 Kohnodai, Ichikawa, Chiba 272-8516, Japan

3 Pathology Division of Clinical Laboratory, National Center for Global Health and Medicine, Tokyo, Japan

4 Department of Gastroenterology and Hepatology, National Center for Global Health and Medicine, Tokyo, Japan

5 Present Address: Department of Gastrointestinal Tract Surgery, Fukushima Medical University School of Medicine, Fukushima, Japan 
Fig. 1 Kallikrein-related peptidase 13 (KLK13) expression in esophageal squamous cell carcinoma (ESCC) was lower than that in normal esophageal mucosa. a KLK13 transcript levels determined with revere transcription-polymerase chain reaction in paired samples from 24 patients with ESCC. Data indicate expression relative to the mean levels of normal tissues. b Immunohistochemical analysis of KLK13 expression in noncancerous tissue and ESCC specimens. Representative staining of KLK13-negative or KLK13-positive samples

\section{A}

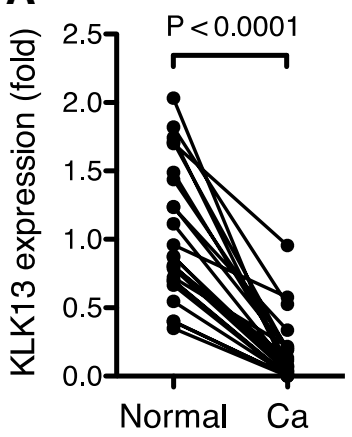

B Normal

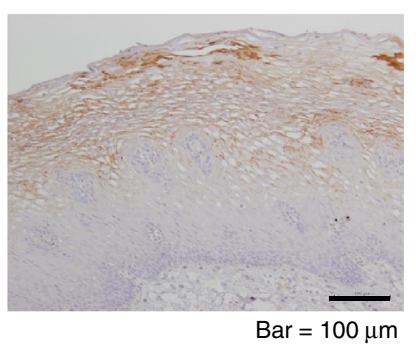

ESCC (KLK13 negative) ESCC (KLK13 positive)
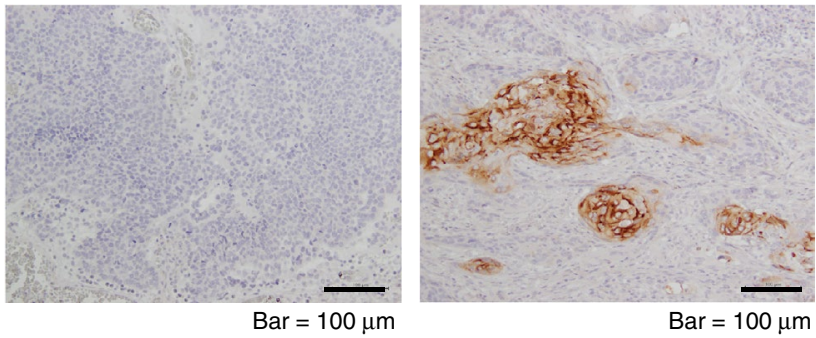